\title{
Improvement of Thermal Comfort Conditions in an Urban Space (Case Study: The Square of Independence, Sétif, Algeria)
}

\author{
By Ballout Amor ${ }^{1}$, Dhia Eddine Zakaria Lacheheb ${ }^{2}$, Yasmina Bouchahm ${ }^{3}$
}

\begin{abstract}
Several studies all around the world were conducted on the phenomenon of the urban heat island, and referring to the results obtained, one of the most important factors that influence this phenomenon is the mineralization of the cities which means the reducing of evaporative urban surfaces, replacing vegetation and wetlands with concrete and asphalt. The use of vegetation and water can change the urban environment and improve comfort, thus reduce the heat island. The trees act as a mask to the sun, wind, and sound, and also as a source of humidity which reduces air temperature and surrounding surfaces. Water also acts as a buffer to noise; it is also a source of moisture and regulates temperature not to mention the psychological effect on humans. Our main objective in this paper is to determine the impact of vegetation, ponds and fountains on the urban microclimate in general and on the thermal comfort of people along the Independence square in the Algerian city of Sétif, which is a semi-arid climate, in particularly. In order to reach this objective, a comparative study between different scenarios has been done; the use of the Envi-met program enabled us to model the urban environment of the Independence Square and to study the possibility of improving the conditions of comfort by adding an amount of vegetation and water ponds. After studying the results obtained (temperature, relative humidity, PMV and PPD indicators), the efficiency of the additions we've made on the square was confirmed and this is what helped us to confirm our assumptions regarding the terms of comfort in the studied site, and in the end we are trying to develop recommendations and solutions which may contribute to improve the conditions for greater comfort in the Independence square.
\end{abstract}

Keywords: microclimate, vegetation, water ponds, simulation, Envi-Met software, comfort.

\section{Introduction}

The treatment of outdoor spaces in urban areas helps mitigate the roughness of climatic stresses around buildings and in public spaces to make these spaces more comfortable to live and the longest time possible (Robitu, 2005).

To reach this comfort, the use of certain urban planning is essential. Among the most effective and most widely used improvements, we can mention the use of vegetation and water bodies, especially when they are associated with one another.

Water and vegetation play a major role in creating a more comfortable microclimate in outdoor spaces and around buildings and, and they come alone to solve many problems in urban areas (Nikolopoulou, 2004).

The vegetation has an effect on the overall urban climate and microclimate in public areas and around buildings. In addition to its aesthetic appearance, urban vegetation promotes social activity, reduces the impact of noise pollution, offers seasonal shade, acts as a screen against wind, cools the air through evapotranspiration, filter suspended dust and regenerate air by producing oxygen (Liébard \& De Herde, 2006).

${ }^{1}$ University of Hadj Lakhdar, Batna, Algeria

2 Work group PUVIT, Sétif, Algeria

${ }^{3}$ Work group ABE, Université Mentouri, Constantine, Algeria 
The purpose of the construction of a fountain, a water jet or a pond is not only beautify the environment but also to cool it by evaporation, to mask the traffic noise, helps people in relaxing and provide solutions to thermal control of space, in hot climatic situation (Leguay, 2002).

Ponds and fountains can be efficient air-conditioning in open spaces because of their ability to maintain water temperatures below the temperature of the air, and their low reflectivity. As the water evaporates, its temperature decreases and this evaporation is proportional to the surface of air / water contact (Santamouris \& Adnot, 2004).

After having discussed the role of vegetation and water in the city, it is interesting to note that the combination of water and vegetation seems inseparable or at least represents the best complementarily in urban environment.

In order to demonstrate the important role of the presence of water and vegetation in creating a favorable microclimate a case study will be taken as an example and which is: the Independence Square (Square of Ain-El-Fouara), urban area located in the town of Setif(Algeria), characterized by the presence of a water fountain, and surrounded by a large plant mass.

\section{Objectives}

In this work, we are looking to achieve the following objective:

* -Evaluate and quantify the impact of the vegetation and the fountain on thermal comfort in our case study.

* -Proceed to a calibration of results obtained through the simulation by comparing them with the results of the investigation.

* -In order to further improve the comfort in the square and upgrade the urban space, thanks to a kind of projection into the future and that thanks to the simulation.

* -Demonstrate that thermal regulation of urban microclimate in areas with hot dry climate is possible by a judicious choice of size, disposition and amount of vegetation and water bodies.

\section{Methodology}

Two methods were used to reach the objectives of this paper; the first concerns the application of a field investigation with a series of measurements on the factors affecting thermal comfort in Independence square. The investigation provides operational and practical tool that allow acquiring observable, quantifiable and close to reality.

The second is a simulation, based on computer software, allowing accurate quantification of micro-climatic impacts related to the parameters of the vegetation and water on the outdoor thermal comfort, but also to simulate several scenarios with different feature of the place.

A comparison of the measurements with the result of the simulation performed by the Envi-Met software will be made considering the square with the current state. This comparative study appears to be useful to the extent that it allows us to determine whether a relationship exists between the two types of data, but also to check the existence of anomalies in the model simulated.

Another comparison between the results of the five scenarios with different feature of the square, allowing a quantification of the impacts of vegetation and water on the outdoor thermal comfort. We will try to conclude at the end of our work and try to suggest some recommendations and solutions with reference to the results later.

\section{Intervention Site}

The choice of site for this study concerns the Independence square, one of the most important and best known urban spaces of the city of Sétif, which had a semi arid climate. 
In addition, this place is a mineral open space with a water source and abundant vegetation, will give us the opportunity to test our hypothesis about the role of these two combined elements in terms of microclimatic comfort.

The Independence Square is characterized by a rectangular form $(70 \mathrm{mx} 80 \mathrm{~m})$, corresponding to the general layout of the city.

Its location in the city and its historical identity influences directly its attendance.

The vegetation is abundant and the fountain of Ain el Fouara which has a symbolic value occupies the center of the square, which should provide more comfort and convenience for users, and protect them from intense solar radiation and contribute to the temperature reduction, and humidifying the air.

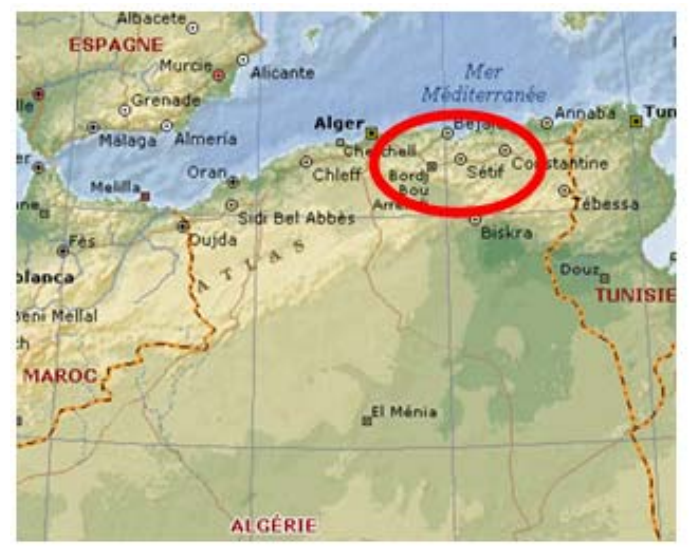

Figure.1: Location of the town of Sétif (Algeria). (Source: Encarta, 2006)

\section{Interpretation and Analysis of the In- Situ and Simulation Measurements}

\subsection{The measurement campaign}

The measures were taken on the $1^{\text {st }}$ August 2007, each hour from 6:00 am to 8:00 pm, which provides the values of all climatic parameters measured (Air temperature, Relative humidity and wind

speed), in four (04) points distributed in the square.

Each point has different physical characteristics than the others, and the classification criteria were the presence of vegetation, the proximity to the water and the exposure to sunlight.

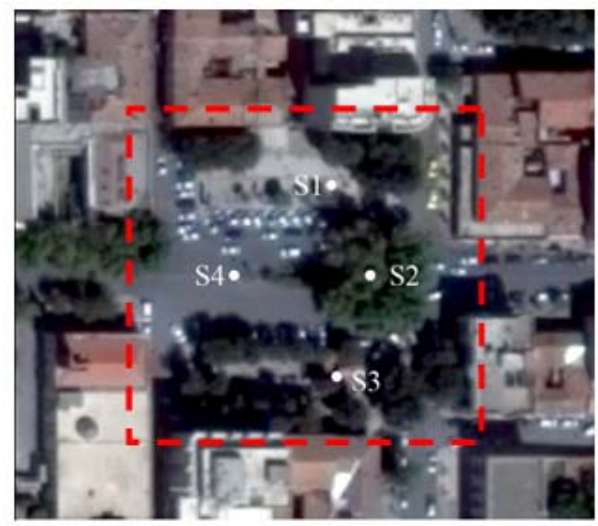

Figure.2: The four stations and their position in the square. (Source: Google Earth)

\subsection{Conclusion of the measurement campaign:}

The measurement campaign carried out in the square of independence, allowed us to confirm the microclimate character of the place. Whether from the spatial perspective (different parameters relating to thermal comfort between the four areas) but also throughout time, because the thermal characteristics of each station change depending on the time of day. The station S3 is the most fresh station early in the day, while the station S2 becomes the freshest one at the end of the day, the station S4 is the hottest station throughout the day with a maximum of up to $3.7^{\circ} \mathrm{C}$ between that station and the station S2. 


\section{The Simulation}

The simulation was conducted to verify and consolidate the results obtained during the investigation, but also to identify parameters that could brings new elements to our research. In our study, the two parameters studied are the presence of water and the density of plant cover, and to see if the square as it is now (with the fountain and the existing trees) provides the optimal conditions of comfort, or we could further improve the comfort in this area by adding water surfaces and even more trees.

The physical parameters studied were air temperature, relative humidity, and wind speed, other physiological parameters are also used as the PMV index (Predicted Mean Vote) and the PPD index (Predicted Percentage dissatisfied).

\subsection{Comparison between the measured and the simulated values}

This comparative study between measured and simulated values is useful since it allows us to establish a relationship between the two types of data, but also to verify the existence of anomalies in the simulated model. We would like to clarify that this comparison is made between the values recorded in-situ and those of the second scenario which is the square as it exists in reality.

We also note that in this comparison we use the mean value recorded in the four stations both for the measured or simulated values, and are referred to as now as average temperature of the square, average relative humidity of the square, etc.

\subsubsection{Temperature}

Globally the two curves go through two major periods, the first period were the two curves increases to reach a maximum value $\left(37.5^{\circ} \mathrm{C}\right.$ at $1: 00 \mathrm{pm}$ to the measured temperatures and $36.38^{\circ} \mathrm{C}$ at 2:00 pm for simulated ones). A second period in which the two curves decreases to reach their minimum at the end of the period of investigation.

It is clear that the simulated temperature curve is more regular than the measured one which is more random. This can be explained by anthropogenic inputs due to traffic but also to the large number of individuals present in the square during rush hour (precisely 8:00 am, 1:00 pm and 5:00 $\mathrm{pm}$ ). These contributions are not considered by the software and that is why the simulated curve is as perfect as that.

The gap between those simulated and measured temperatures can reach $1.9^{\circ} \mathrm{C}$ at $8: 00 \mathrm{am}$ and $2.71^{\circ} \mathrm{C}$ to $4: 00 \mathrm{pm}$; this is due to anthropogenic inputs mentioned above.

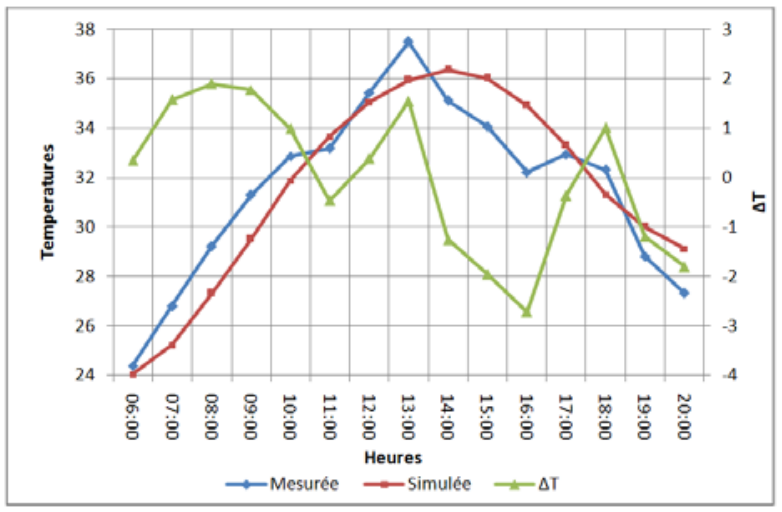

Published by ECSDEV, Via dei Fiori, 34, 00172, Rome, Italy
Figure.3: Comparison of measured and simulated temperatures. (Source: author)

\subsubsection{Relative humidity}

The curve of simulated humidity is more regular than the measured humidity, and this is always due to anthropogenic and katabatic (relative wind) inputs that exist in reality and which are not considered by the ENVI-met 
software. For the measured values, we note that there are sudden drops in relative humidity during peak hours $(8: 00 \mathrm{am}, 1: 00 \mathrm{pm}$ and $5: 00 \mathrm{pm})$ which can only be answers to temperature increases during these critical hours.

Differences can reach $9.14 \%$ at $8: 00 \mathrm{am}$ and $7.65 \%$ at $5: 00 \mathrm{pm}$ and a minimum value of $0.15 \%$ at 11:00am, with an average difference of $4.5 \%$. After consulting a number of studies dealing with the thermal simulation in urban areas, we note that there are still differences between the simulated values and those measured and the recorded differences in our case is quite acceptable.

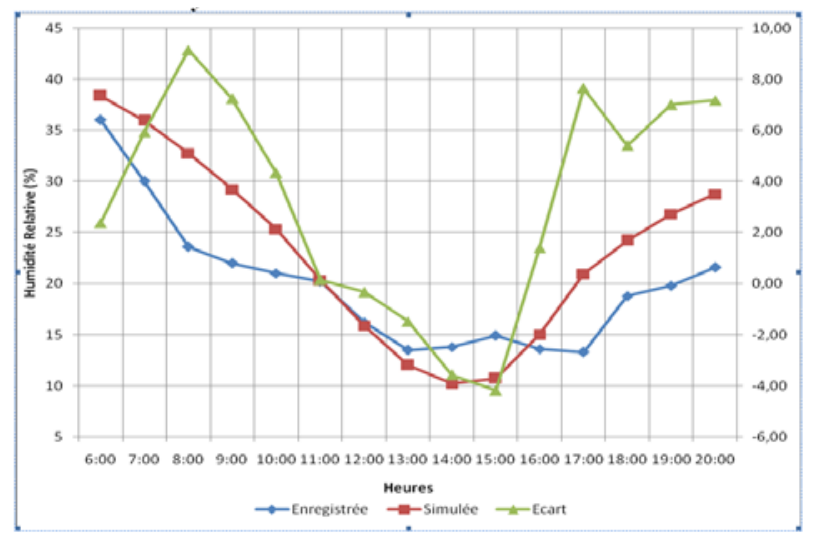

Figure.4: Comparison of measured and simulated Relative bumidity. (Source: author)

\subsubsection{Wind speed}

The curve of the simulated wind speeds is more regular than those recorded. This is mainly due to the ENVI-met software, which reached its limits in terms of simulation model and uses a too regular model for a parameter as random as the wind. Overall, the simulated values show that the square is not very windy throughout the day with a slight fall of $0.1 \mathrm{~m} / \mathrm{s}$ in the early afternoon, however, the curve is quite stable. The values recorded show that there is some instability of wind speed throughout the day with a slight increase in the late afternoon.

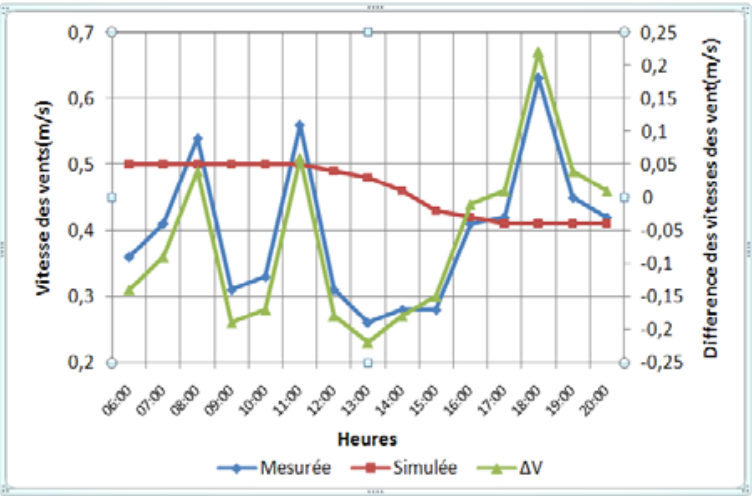

Figure.5: Comparison of measured and simulated values of wind speed. (Source: author)

\subsubsection{General deduction}

It can be inferred from the comparison between the measured values and those simulated with the ENVI-met software, that the relationship between simulated and measured values is quite logical, however it is clear that there are differences between those two types of values but that are tolerable, this difference changes depending on time of day.

After this step we move to the comparison of the five scenarios and these results will be compared, and it would be interesting to see the evolution of different physical parameters (temperature, humidity and wind speed) and physiological (PMV and PPD). 


\section{Effects of Arrangements on the Parameters Related to Comfort in the Square}

To show the effect of the different arrangements on the comfort parameters, whether the physical parameters (temperature, humidity and wind speed) or physiognomic (PMV and PPD), we compared the values of the five.

We took as an example the measurements stored in station S2 in which the improvements have made the best results.

\subsection{Presentation of the five scenarios}

\subsubsection{Scenario $\mathrm{N}^{\circ} 1$}

In this scenario there is no vegetation and no water surface. This means that the simulation is designed to check the parameters for thermal comfort of the square in the initial state with no elements that could improve thermal comfort.

\subsubsection{Scenario $\mathrm{N}^{\circ} 2$}

This scenario corresponds to the current state of the square, which is to say the existence of the fountain in the middle and vegetation that is equivalent to that present in reality. In terms of surface that represents $16.3 \%$ of the total area of the square.

\subsubsection{Scenario $\mathrm{N}^{\circ} 3$}

In this scenario have been preserved the same vegetation density than the second scenario $(16.3 \%$ of the total area), and have been added a water surface which corresponds to the median strip existing in the middle of the square, which surface is $214 \mathrm{~m}^{2}$ or $3.7 \%$ of the total area of the square.

\subsubsection{Scenario $\mathrm{N}^{\circ} 4$}

We kept the same vegetation density as the third scenario, and two pool was added to the left and right of the fountain in addition to that added in the 3rd scenario, corresponding to a total surface of $770 \mathrm{~m}^{2}$ or $1334 \%$ of the total area.

\subsubsection{Scenario $\mathrm{N}^{\circ} 5$}

In this scenario we have kept the same surface of water ponds $(13.34 \%$ of total area), while the density of vegetation is increased to $34.92 \%$ of the total area of the square.

\subsection{Effects of various arrangements for station S2:}

It was noted that the station S2 records the most important improvements and that's why we will focus on this station to show how far we can enhance the outdoor comfort (in our case study) by using both passive solutions.

This fact can be explained by the fact that the station S2 is below four old trees that have a very dense crown (12 $\mathrm{m}$ diameter) and closely spaced, which amplifies the effect of shadows projected and the effect of evapotranspiration and thus contributes to a greater fall of temperature, unlike the other stations located near sparse vegetation.

\subsubsection{On the air temperature}

The first thing you notice is that the temperature recorded in the station S2 decreases gradually as we added improvement. But it is clear that this diminution is different from a scenario to another.

The curves of the five scenarios, follow the same pace, i.e. they start from a minimum value early 
in the day. Then they rise gradually to a maximum value at 1:00pm or 2:00pm, and finally decrease gradually and reach a minimum value at the end of the simulation.

Every time we added an improvement the air temperature decreases. We noted that the first addition of vegetation seems to cause the largest drop with an average of $1.31^{\circ} \mathrm{C}$. The addition of the first pond lowered the temperature to an average of $0.20^{\circ} \mathrm{C}$ (with a max of $0.32^{\circ} \mathrm{C}$ at $6: 00 \mathrm{am}$ and a min of $0.8^{\circ} \mathrm{C}$ to $\left.1: 00 \mathrm{pm}\right)$.

The addition of the two ponds seems to record the less important drop of temperature with an average of $0.09^{\circ} \mathrm{C}$, this is due to the fact that the two ponds are quite far from the station S2.

The latest addition of vegetation decreases the temperature of $0.69^{\circ} \mathrm{C}$ (with a max of 0.81 at 6:00 am and a min of 0.51 to 2:00 pm). The decline is less significant because the addition of the vegetation is performed far from the area of the station S2.

It is noted clearly that the different arrangements made, have contributed to an average drop in temperature in this area of $2.29^{\circ} \mathrm{C}$, the maximum difference is equal to $3.43^{\circ} \mathrm{C}$ at $6: 00 \mathrm{am}$ and the minimum difference is equal to $1.61^{\circ} \mathrm{C}$ at $1: 00 \mathrm{pm}$.

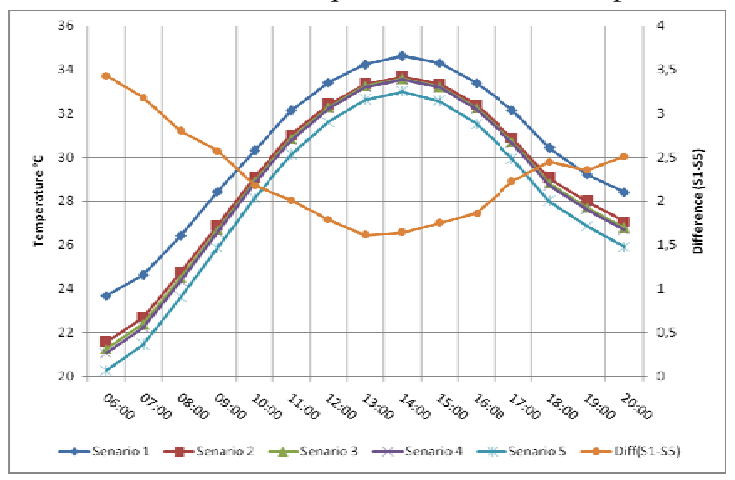

Figure.6: Temperature values recorded for the five Scenarios in the station S2.(Source: author).

\subsubsection{On the relative humidity of the air}

The relative humidity is at its maximum at 6:00am for all scenarios. These values start to decrease until it reaches a minimum at 2:00pm, and finally increase gradually and reach a maximum value at the end of the day.

As for temperature, also we note that for each improvement the result obtained is different.

We noted that the first addition of vegetation seems to cause the largest increase (due to effect of evapotranspiration of the vegetation) with an average of $3,54 \%$, with a maximum increase of $6,46 \%$ at $10: 00$ am.

The addition of the first pond increased the humidity to an average of $1,35 \%$ (due to the evaporation from water ponds), with a max of 3,34\% at 6:00am and a min of $0.9 \%$ to 1:00am). The addition of the two ponds seems to record the less important increase of relative humidity with an average of $0.27 \%$, this also can be explained by the fact that the two ponds are quite far from the station S2.

The latest addition of vegetation increases the humidity of 2,04\% (with a max of 3,81 at 9:00am and a minimum of $1,29 \%$ to $2: 00 \mathrm{pm})$.

For this parameter we can also notice that the combination of the arrangements have contributed to an average increase in humidity in this area of $7,14 \%$, the maximum difference is equal to $11,53 \%$ at $9: 00 \mathrm{am}$ corresponding to a minimum stomatal resistance and the minimum difference is equal to $1.61 \%$ at 1:00am were the thermal stress is at its maximum .

The moisture supplies for this station confirm the importance of the density of vegetation cover, the more the canopy is dense the more the water vapor emitted to the surrounding air is important, in addition to this, moisture will be trapped under the crowns of trees unlike the case of isolated trees. 


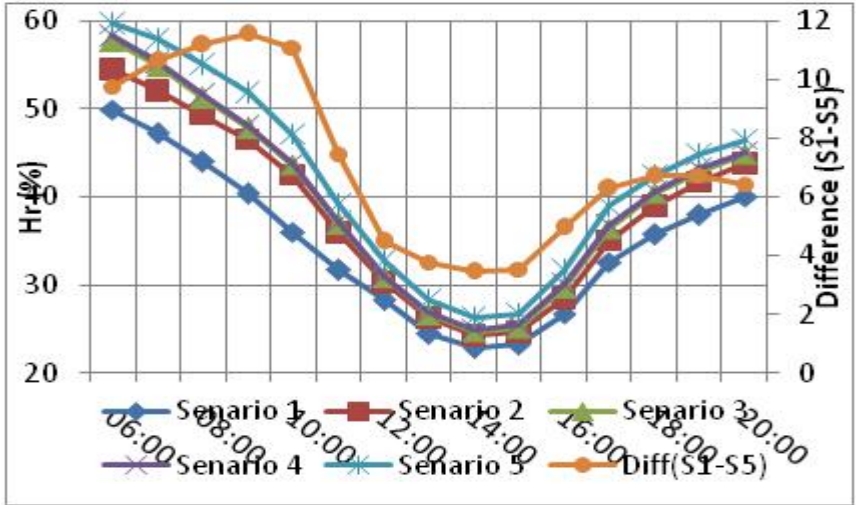

Figure.7: Relative humidity values for the five scenarios in the station $S 2$.

(Source: author).

\subsubsection{On the PMV and the PPD}

We would recall that the PMV is a complicated index dependent of several parameters (air temperature, mean radiant temperature, humidity, air velocity, metabolism and the thermal resistance of clothing) and for this reason the results can be unexpected. But we will try to give a logical explanation for this.

PMV values are at their lowest at 6:00am. These values begin to rise until it reaches the maximum values of the day at 2:00pm. From this time, values begin to decrease gradually to reach the lowest value of this period.

Improvements in square have contributed to an average decrease of PMV of 0.72 , with a maximum variation of 1.21 at 6:00am and a minimum of 0.45 at 2:00pm.

As expected the first addition of vegetation seems to cause the largest amelioration with an average of 0.44 in the PMV value, with a maximum increase of 0.86 at 6:00am. The addition of the first pond decreased the PMV value to an average of 0.11 , with a max of 0.15 at 6:00pm and a min of 0.6 to 9:00am).

The addition of the two ponds seems to record the less important decrease of PMV with an average of 0.03 this also can be explained by the fact that the effect of the evaporation is far from the station S2.

The latest addition of vegetation decreases the PMV of 0.23 (with a max of 0.41 at 6:00am and a minimum of 0.13 to $2: 00 \mathrm{pm}$ ).

In term of periods of comfort, we can say that after each amelioration the curve of PMV values get more and more flatten and this makes the period of comfort extends as the discomfort periods decrease (hot and very hot period). It may be noted for the overall amelioration that the period of comfort extends to 10:00am instead of 9:00am, later it starts at 6:00pm instead of $8: 00 \mathrm{pm}(33 \%$ instead of $20.83 \%$ of the total period). The hot period constitutes $27 \%$ of the total period instead of $30.83 \%$. At the end, the very hot period fits in the interval 12:00am-4:00p.m. instead of 10:35a.m-5:15p.m. (Which means $29.16 \%$ instead $48.83 \%$ of the total period).

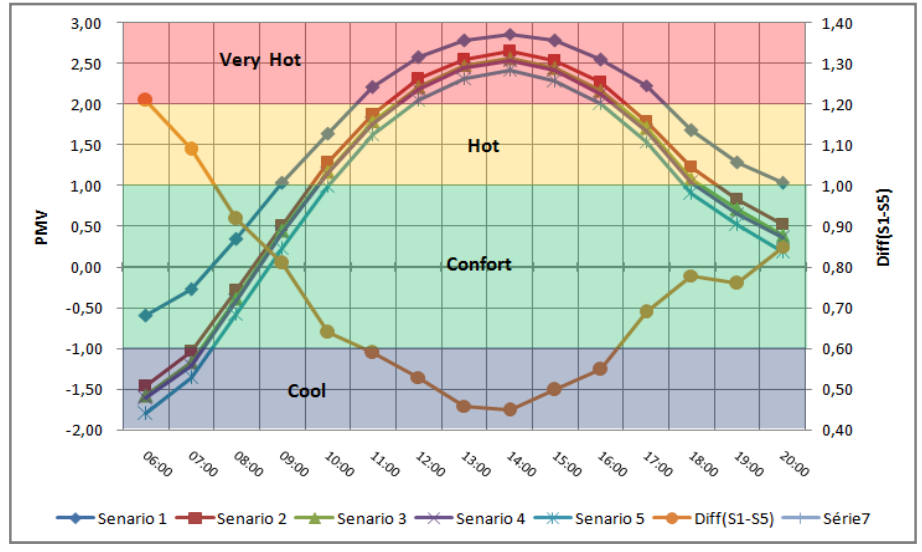

Figure.8: PMV values recorded for the five scenarios in the station S2. (Source: author). 
The curve of differences registers peaks at the beginning and end of the day corresponding to maximum ameliorations (1.21 at 6:00am and 0.85 at 8:00pm) and knows a drop at midday which brings us back to say that the improvements (trees and water ponds) have an optimal effect in both periods, while this effect decrease at midday.

The average decrease of dissatisfaction is $21.99 \%$ (for positive values) with a maximum variation of $38.91 \%$ at $6: 00 \mathrm{pm}$ and a minimum of $7 \%$ to $2: 00 \mathrm{pm}$.

For the first addition of vegetation an average decreases of $21.92 \%$ of dissatisfaction, with a maximum increase of $38.57 \%$ at 6:00am.

The addition of the first pond decreased the PPD value to an average of $3.01 \%$, with a max of $6.67 \%$ at $9: 00 \mathrm{pm}$ and a min of $0.6 \%$ to $14: 00 \mathrm{am})$.

The addition of the two ponds records an average decrease of 0.67 , and the last addition of vegetation decreases the PPD of 5.53\%.

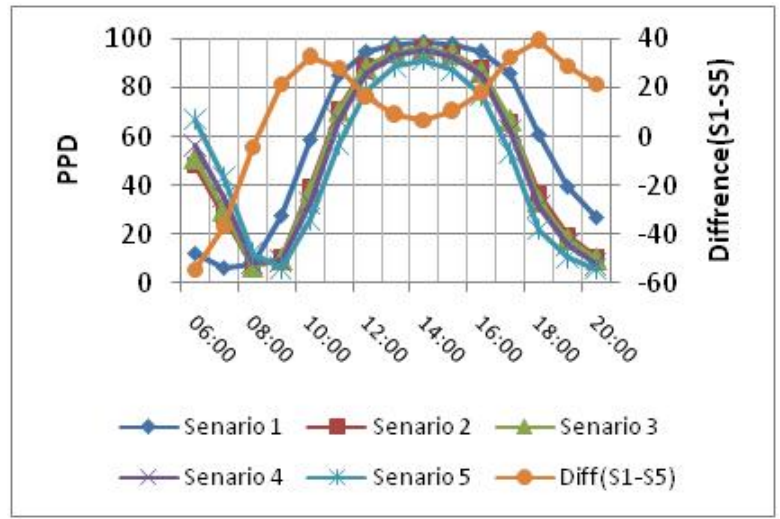

Figure.9: PPD values recorded for the five scenarios in the station S2.(Source: author).

\subsection{Discussion of the differences}

The comparison of the five scenarios was made in order to quantify the effect of each improvement and also the cumulative effect of all improvements on the various parameters modifying thermal comfort in the Independence square.

Regardless of the location of the station in the site a beneficial contribution of the different facilities is clearly quantified. In terms of thermal comfort of pedestrians and cooling efficiency of the vegetation and water led to a number of conclusions, and the most important are:

*-The largest differences were recorded at the beginning and end of the day, unlike the mid-day when heat stress is so important that the effect of the improvements is minimal.

*-The effect of the density of vegetation on different parameters of comfort seems to be more efficient than the effect of water ponds.

*-Lowering temperature can reach $1.64^{\circ} \mathrm{C}$ at $2: 00 \mathrm{pm}$ (where the thermal stress is maximal) for overall improvement, where the addition of vegetation (scenario 1 and 5) can lower $1.53^{\circ} \mathrm{C}$ on its own.

*-Relative humidity registers a maximum increase of $3.44 \%$ at 2:00pm for overall improvement, vegetation can rise the amount of relative humidity by $2.79 \%$. The overall difference is directly linked to the stomatal resistance of leafs.

*-The PMV is a physiological interpretation of these parameters; it is also registering an improvement of up to 0.45 to $2: 00 \mathrm{pm}$ corresponding to a lower dissatisfaction rate of $7 \%$.

*-The PMV are directly affected by the stomatal resistance of the leafs and this is clearly noted in the difference of the 1st and 5th scenarios on one hand and the 2nd, 3rd and 4th scenarios on another hand. Wind speed also affects the PMV.

*- The period of comfort may represent $30 \%$ of the simulation period with the improvements instead of $23.83 \%$ (around the station S2), while periods of discomfort where significantly decreasing like the very hot period decreased from $48.83 \%$ to $29.16 \%$ which equals to almost three hours long. 


\section{Conclusion}

First, we can easily confirm that the relationship between simulated and measured values is logical and this despite the differences that exist between the two values but still remains quite reasonable.

This allow us to continue on the second step which is the comparison between the different scenarios and verify the role of different levels of the plant cover and evaporative surfaces (water bodies) in improving the thermal comfort conditions in urban areas.

The simulation allowed us a kind of projection into the future, checking different scenarios we should have wait several years and spent large sums of money to be in the ability to verify the microclimatic characteristics.

We tried to investigate the relationship between the presence and density of vegetation and surface evaporative (water ponds) on one hand and thermal comfort in urban outdoor space, on the other hand.

The improvements due to the effect of vegetation in urban outdoor space are susceptible and this by the creation of sources of freshness. This vegetation acts on the quantity of transmitted radiation, air speed, the mean radiant temperature, surface and air temperatures as well as the specific air humidity. These changes generate improvements in thermal comfort and this benefic effect on comfort it is more important for dense vegetation than sparse one.

We can retain that the effect of the water ponds is very located on the thermal comfort. The water ponds have a local influence on the specific humidity of the air and to a lesser degree on temperature. However we must not forget that the only parameter taken into account is the area of evaporative ponds, other parameters could be studied or simulated such as the height of one or several water jets, the height of a waterfall, the flow of the water pond or even misting or brumisation which still remains a more complex process than a jet of water or a fountain. And this of course with other programs other than ENVI-met which does not offers these opportunities and had as unique configuration a standing deep water.

While the temperature and humidity are purely physical value, the PMV and the PPD are at the same time physiological and quantitative and allows us to better understand the evolution of the comfort throughout time (the simulation period all over the day) and also at each improvement that is applied in the square.

Other parameters could be tested (nature of materials, vegetation, depth of the water pool etc.). These additional studies can help to give the rules of assistance in the design facilities like water ponds and vegetation.

\section{References}

Nikolopoulou, M. (2004): Concevoir des espaces extérieurs en environnement urbain : une approche bioclimatique. Center for Renewable Energy.

Liébard, A. \& De Herde A. (2006): Traité d'architecture et d'urbanisme bioclimatiques. Edition : Le Moniteur. Leguay, J.-P. (2002): L'eau dans la ville au Moyen âge. Presses universitaires de Rennes.

Santamouris, M. \& Adnot J. (2004): Cooling the cities. Edition: Presses de l'École des Mines de Paris.

Robitu, M. (2005): Etude de linteraction entre le batiment et son environnement urbain : influence sur les conditions de confort en espaces extérieurs. Thèse de doctorat, Nantes: Université de Nantes. 